\title{
Tendencias en la financiación pública de la Educación Superior
}

\author{
Joan Rosselló Villalonga \\ Universidad de las Islas Baleares
}

\section{Resumen}

En este artículo se analizan los efectos de los modelos de financiación de las universidades públicas sobre la calidad de la oferta educativa. La primera parte se centra en el modelo de financiación español y sus efectos sobre la estratificación de las universidades (aparición de universidades de élite) y la segregación de los estudiantes por su talento. En la segunda parte, se analizan las reformas que se están llevando a cabo en distintos países de la OCDE, inspirados en el modelo de provisión pública de enseñanza superior que funciona actualmente en Estados Unidos. Algunos aspectos de estas reformas podrían permitir corregir ineficiencias que caracterizan el modelo de financiación universitaria en España.

Palabras clave: financiación de la educación superior, estratificación universidades, segregación estudiantes, reforma educativa.

Clasificación JEL: I22, I23, I28

\begin{abstract}
This article analyzes the effects of public universities funding systems on the quality of the education offered. The first part of the paper focuses on the Spanish funding model and its effects over the stratification of universities (the emergence of elite universities) and the segregation of students according to their talent. In the second part of the article, we analyze the reforms that several OCDE countries are carrying out, inspired by the public provision model of university education that is currently in place in the United States. Some aspects of these reforms could allow to correct the inefficiencies that characterize the university funding model in Spain
\end{abstract}

Keywords: finance of higher education institutions, universities'stratification, students'segregation, educational reforms

JEL Classification: I22, I23, I28

\section{Introducción}

Desde hace algunos años los países de la Unión Europea se están planteando un profundo proceso de reformas en sus respectivos modelo universitarios (ver Mas-Colell 2003), tal y como se acordó en la Conferencia de Lisboa (2000).

Este planteamiento nace de la crítica que ha recibido el modelo de universidad pública de los países europeos por su escasa capacidad de generar el suficiente nivel de 
capital humano y de resultados de inversión en I+D para competir con economías como la de Estados Unidos, Japón o más a largo plazo con China ${ }^{1}$. Las causas abarcan desde la escasez de recursos que se destina a la educación superior (1.1 del PIB en la UE frente al $2.7 \%$ en EEUU o los datos en términos per capita los cuales muestran que España está 28 puntos por debajo del gasto per cápita en EE.UU), la excesiva dependencia financiera de las universidades respecto de las transferencias de las AA.PP, la falta de autonomía en la gestión por parte de las propias universidades (en cuanto a criterios de selección de los estudiantes, contratación de profesorado, sistemas de incentivos a la investigación), la política de dispersión de los recursos destinados al fomento de actividades de I+D, etc.

TABLA 1

GASTO POR ALUMNO EN ENSEÑANZA SUPERIOR EN RELACIÓN AL PIB PER CÁPITA

\begin{tabular}{|l|c|c|c|c|c|c|}
\hline \multirow{2}{*}{} & \multicolumn{7}{|c|}{ Ã NO } \\
\cline { 2 - 6 } & $\mathbf{1 9 9 7}$ & $\mathbf{1 9 9 8}$ & $\mathbf{1 9 9 9}$ & $\mathbf{2 0 0 0}$ & $\mathbf{2 0 0 1}$ & $\mathbf{2 0 0 2}$ \\
\hline Alemania & 43,0 & 41,0 & 42,0 & 42,0 & 41,0 & 41,0 \\
Canadá & 62,0 & 58,0 & 57,0 & 43,0 & - & - \\
España & $\mathbf{3 2 , 0}$ & $\mathbf{3 0 , 0}$ & $\mathbf{3 0 , 0}$ & $\mathbf{3 3 , 0}$ & $\mathbf{3 5 , 0}$ & $\mathbf{3 5 , 0}$ \\
Estados Unidos & 59,0 & 61,0 & 57,0 & 59,0 & 63,0 & 57,0 \\
Finlandia & 35,0 & 34,0 & 35,0 & 33,0 & 42,0 & 42,0 \\
Francia & 34,0 & 34,0 & 34,0 & 33,0 & 33,0 & 34,0 \\
Grecia & 29,0 & 29,0 & 27,0 & 21,0 & 25,0 & 25,0 \\
Irlanda & 39,0 & 38,0 & 37,0 & 39,0 & 34,0 & 30,0 \\
Portugal & - & - & 28,0 & 28,0 & 29,0 & 37,0 \\
Reino Unido & 40,0 & 45,0 & 41,0 & 39,0 & 40,0 & 41,0 \\
Media OCDE & $\mathbf{4 5 , 0}$ & $\mathbf{4 4 , 0}$ & $\mathbf{4 4 , 0}$ & $\mathbf{4 2 , 0}$ & $\mathbf{4 2 , 0}$ & $\mathbf{4 3 , 0}$ \\
\hline
\end{tabular}

A pesar de que la necesidad de reformas transciende el ámbito financiero, será en éste donde a medio plazo deberán realizarse profundos cambios. Las crecientes necesidades financieras de las universidades públicas, producto de una mayor demanda en la prestación de servicios, más allá de los ligados estrictamente a la educación superior (formación continua, inversión en I+D+i etc.), para financiar la inversión en capital físico y humano, no parece que vayan a cubrirse exclusivamente, como ha sucedido hasta ahora, con recursos procedentes de las Administraciones, debido en parte a las propias restricciones presupuestarias de éstas. Un segundo problema con el

1 Ver The Economist, 8 de septiembre 2005. 
modelo actual de provisión pública de la educación es que genera ineficiencias, por la falta de incentivos, sobre los resultados de la gestión de las universidades así como sobre los propios estudiantes (en cuanto a sus resultados académicos, etc.)

De ahí la tendencia a que las universidades dediquen cada vez mayores esfuerzos a captar recursos del sector privado, tanto desde el sector productivo (recursos ligados básicamente a la investigación y asistencia técnica) como de los propios estudiantes (tasas y matrículas).

Esta problemática también ha recibido atención desde el ámbito de análisis teórico. En concreto, actualmente se trabaja en dos líneas ligadas al tema de la financiación de la educación superior. A pesar de la complementariedad entre ambas líneas de investigación, es importante destacar la necesidad de separar la financiación de las universidades de la financiación de los estudios a los beneficiarios. Una reforma en el sistema de financiación de las universidades no tiene por qué afectar la decisión de los futuros estudiantes si se diseña un sistema de ayudas que permita posponer el pago de la educación recibida al momento de la finalización de los estudios.

De acuerdo con esta distinción, en la literatura encontramos una línea de investigación en la cual se analizan los efectos de distintos modelos de financiación de las universidades - elección óptima de precios públicos, tasas, etc- sobre el número de matriculados y su bienestar (ver Del Rey y Romero (2004), Ehrember y Sherman (1984), Ehremberg y Rizzo (2004), Fethke (2005), Golding y Katz (1998), Greene (1994) y Koshal y Koshal (2000), entre otros), sobre la calidad de la enseñanza que ofrecen las universidades y sobre la generación del output científico (ver De Fraja y Iossa (2002), Del Rey (2001), Beath et al (2003) y Rosselló (2006)), en un entorno competitivo, tanto entre universidades como entre administraciones.

Una segunda línea analiza el diseño óptimo de sistemas de ayudas y becas, que permitan a los estudiantes hacer frente a unas tasas y matrículas más elevadas sin que éstas supongan una barrera al acceso a la formación superior (ver Cigno y Luporini (2003), Del Rey y Racionero (2006), Epple, Romano y Siegh (2003), García-Peñalosa y Walde (2000), Kemnitz (2004) y Rose y Sorensen (1992), entre otros).

Este tipo de aportaciones adquieren su relevancia en un momento en que países como Reino Unido, Australia y Canadá (por citar algunos de la OCDE) llevan a cabo distintas reformas en sus modelos de financiación de las universidades públicas, orientadas, al igual que ya sucede en Estados Unidos, a permitir a la universidades un mayor grado de flexibilidad en la fijación de las tasas — por lo tanto ingresos- que pueden cargar sobre los estudiantes, permitiendo a su vez que éstas compitan entre ellas en precios. Esto es, se apuesta por trasladar una mayor porción de los costes de la educación superior desde las administraciones a los beneficiarios. Todo ello acompañado de una mejora en el sistema de becas y ayudas a los estudiantes.

En este sentido, la actual reforma de la universidad española podría haberse aprovechado para incluir modificaciones en el modelo de financiación de las universidades, al estilo de lo que ya han hecho algunos de países, y con ello corregir algunas ineficiencias derivadas del modelo actual. Sin embargo, todo parece indicar que de momento las Administraciones Públicas siguen apostando por mantener un modelo de financiación 
en el cual las universidades siguen dependiendo de las transferencias de la administración pública, sin repercutir los costes a los estudiantes y las familias, manteniendo bajas las tasas y matrículas de las universidades públicas. Tasas y matrículas que se caracterizan por su uniformidad, sin discriminar ${ }^{2}$ entre los estudiantes por su capacidad o su nivel de renta - excepto los receptores de becas-, modelo que genera un número considerable de ineficiencias en el comportamiento de los estudiantes.

En particular, en la última reforma, las AA.PP españolas han acordado seguir subvencionando los nuevos estudios de grado, cuyas matrículas seguirán muy por debajo del coste real de los estudios. De hecho, esto supone una merma de recursos de las universidades, puesto que los títulos no oficiales (con matrículas libres) como masters y postgrados eran una fuente de financiación de la universidad que ahora se reduce, al convertirse en títulos oficiales, con tasas y matrículas más bajas.

En este artículo analizamos distintos aspectos de las posibles distorsiones que genera el actual modelo de provisión de la enseñanza superior en España. En particular, analizamos los efectos de este modelo de financiación en cuanto a la estratificación de las universidades por su calidad, así como las limitaciones que las restricciones financieras de las propias administraciones generan sobre la calidad que puede ofrecerse en el conjunto del sistema.

El tema es especialmente relevante por cuanto las externalidades derivadas de la educación superior dependen no sólo del número de estudiantes, sino también de la calidad de los estudios, de la adecuación de la oferta a las necesidades del mercado de trabajo, etc.

Aunque la implantación de un modelo de universidad que funciona en un país no tiene por qué funcionar en otro, en este artículo analizamos aquellos aspectos de las reformas que se están llevando a cabo en distintos países de la OCDE (como Australia, Reino Unido o Canadá), inspirados en el modelo de provisión pública de enseñanza superior que funciona actualmente en Estados Unidos que, de aplicarse, podrían permitir corregir algunas de las ineficiencias que caracterizan el modelo de financiación universitaria en España.

A pesar de que las universidades públicas en EE.UU también dependen en gran medida de las transferencias públicas, los estudiantes en esas universidades contribuyen en mayor medida a cubrir el coste de la educación superior. En EE.UU la cobertura por parte de los estudiantes está alrededor del 20\% de media en las universidades públicas ${ }^{3}$. En cambio, en España los ingresos por tasas y precios públicos solamente representan un $16.7 \%$ (datos agregados para el conjunto de universidades en 2000) de los costes totales de las universidades ${ }^{4}$.

Sin embargo, para compensar los efectos negativos que las elevadas tasas podrían tener sobre el número de matriculados, el sistema se acompaña de un sistema de

\footnotetext{
Sí hay cierta diversidad por tipo de estudios, pero depende de cada comunidad autónoma.

Todavía muy lejano de la cobertura en las universidades privadas que está alrededor del $50 \%$.

4 Dicho de otra forma, los ingresos por tasas y matrículas de los estudiantes en 2000 representaban solamente un 17.29 de los ingresos de las universidades (exceptuando operaciones financieras).
} 
becas y ayudas muy completo. Sistema que ha ido complementándose con una serie de ayudas, como los créditos a los estudiantes o beneficios fiscales. De esta forma se consigue que el nivel inicial de renta de los estudiantes-familias no influya a la hora de tomar la decisión de asistir a la universidad, puesto que el pago de los estudios se dilata hasta el momento de haberlos finalizado (y tener ingresos suficientes para pagarlos). Igualmente, el sistema excluye a los estudiantes con recursos financieros y con escaso talento (que deberán cubrir el coste en su integridad) a diferencia de lo que sucede en un modelo con matrículas uniformes y subvencionadas).

Este tipo de ayudas están todavía en una fase embrionaria en los países europeos, quizá con la excepción del Reino Unido, que comienza a aplicarlos de forma extensiva después de haber realizado una reforma en la cual se ha permitido que las universidades realicen incrementos substanciales en las matrículas que pueden fijar sobre los estudiantes. En España, en cambio, en 2006 el presupuesto para becas (agregando todos los ciclos) fue de 570,8 millones de Euros, mientras que la partida reservada para ofrecer créditos a los estudiantes (en todos los ciclos) sólo alcanzó los 0.897 millones de euros (Datos del MEC)

Sin embargo, el modelo de universidad de Estados Unidos no está exento de problemas. Temas como la segregación de los estudiantes por niveles de renta (Hoxby (1997), De Fraja y Iossa (2002), Bowen et al (2005), Epple y Romano (2003) y Cook y Frank (1993)), la baja calidad de la educación ofrecida en muchas universidades públicas (Winston y Zimmerman (2000), Winston (1999)) los problemas derivados de la «marketización» de las universidades (Bok (2003)), el problema de las dificultades para acceder a la información por parte de los estudiantes en un mercado muy segmentado (Avery y Hoxby (2004), McPherson y Saphiro (1998)), o los problemas de fracaso en los estudios universitarios o el abandono de los mismos (College Board (2005b)), son objeto de análisis de los investigadores en Estados Unidos.

En la Sección 2, analizamos las características del modelo universitario español, centrándonos en los efectos del actual modelo de financiación sobre la estratificación de las universidades y sobre la calidad de la oferta educativa. En la Sección 3, se discuten las distintas políticas que se aplican en algunos países anglosajones. Finalmente, en la Sección 4, presentamos las conclusiones.

\section{Eficiencia y equidad en el modelo de financiación pública de la educación superior en España: ¿cantidad vs calidad?}

Uno de los objetivos de la provisión pública de la enseñanza superior es permitir el acceso a la universidad al mayor número posible de estudiantes, posiblemente para potenciar las externalidades derivadas de disponer de un mayor volumen de población con educación superior. El argumento (ver Chatterton Goddart 2003) se basa en los efectos dinámicos que genera la enseñanza superior en el desarrollo del tejido productivo de la región (desarrollo de nuevos sectores productivos, mejoras en los procesos 
de producción en sectores tradicionales, etc.), a través de los efectos sobre el capital humano (en sus dos vertientes, educación y formación). A ello hay que sumar el efecto directo que la universidad tiene sobre la economía regional, como un actor más en el mercado. De ahí el interés de los gobiernos regionales en financiar nuevas universidades en sus jurisdicciones.

En España, la política de matrículas bajas y de expansión en el número de universidades a lo largo de todo el territorio ha contribuido significativamente a incrementar el porcentaje de la población con estudios superiores, con porcentajes incluso por encima de la media de la $\mathrm{OCDE}^{5}$.

La pregunta que cabe hacerse es si una política de revisión al alza de las tasas, incluso fomentando la competencia entre las universidades, buscando incrementar los recursos de las mismas así como corregir algunas ineficiencias, repercutiría en el número de estudiantes. Los datos para algunos países no parecen indicar que el incremento en las tasas académicas afecte negativamente el número de matriculados, ni siquiera a aquellos con menores recursos, poniéndose así en duda uno de los principales argumentos para justificar las tasas subvencionadas. En países como Australia, Nueva Zelanda y Canadá (ver Department for Education and Skills 2003a), el incremento en el volumen de las tasas ha ido parejo a un incremento en el número de matriculados, incluso el tramo de estudiantes con menores recursos. Los datos para Estados Unidos (ver College Board, (2005)) también indican que durante un período en el cual las tasas y matrículas se han incrementado notablemente, la tasa de escolarización de los estudiantes, en todos los percentiles de renta, ha aumentado durante el período 1982-2003.

Una explicación a esta evolución puede ser que el efecto final en el número de matriculados de los incrementos en el coste de la educación superior depende en gran medida del sistema de becas y ayudas y de los ingresos futuros que esperan conseguir con la educación recibida. La restricción presupuestaria en el momento de iniciar los estudios afecta la decisión de los estudiantes sobre si estudiar o no, o a la hora de seleccionar la universidad y los estudios a cursar. Así, puede ocurrir que los estudiantes con restricciones no puedan estudiar o bien que acepten cursar estudios de menor calidad porque tienen precios inferiores ${ }^{6}$.

Pero incluso un sistema de tasas bajas y uniformes ha recibido críticas desde el punto de vista de la equidad, por cuanto puede generar efectos regresivos. Por un lado, porque los beneficiarios en general acabarán teniendo unos salarios superiores a aquellos que no acceden a la universidad ${ }^{7}$. Por otro lado, porque se está subvencio-

5 El porcentaje de la población con estudios superiores en España en el tramo de los 25 a los 34 años ha pasado del $27 \%$ en 1995 al 38\% en 2003, frente a una media de la OCDE del 22 al 29\% y del 34 y $39 \%$ en el caso de Estados Unidos, según datos de la OCDE.

6 Así se ha observado en las matrículas de estudiantes en universidades públicas de ámbito regional en Estados Unidos (ver Bowen et al 2005).

7 Cabe señalar que aunque los trabajadores que han recibido educación superior probablemente tendrán salarios superiores y pagarán mayores impuestos, éstos pagarán los mismos impuestos que cualquier otra persona con el mismo nivel de renta que no haya cursado estudios superiores. 
nando directamente a aquellos con recursos suficientes para financiarse los estudios, al no haber discriminación en las tasas por niveles de renta.

En este sentido, las ayudas al estudio deberían permitir que los estudiantes no estuviesen sometidos a restricciones presupuestarias en el momento de escoger los estudios, sin que ello implique que los estudiantes no cubrirán el coste de los mismos al final, dadas las ineficiencias que se observan en los modelos de tasas subvencionadas.

\subsection{La relación tasas, calidad y esfuerzo en los estudiantes}

Aunque es cierto que en el ámbito universitario la fijación de precios no puede realizarse solamente teniendo en cuenta criterios de oferta y demanda, como en un mercado normal, puesto que hay consideraciones de externalidades, cuestiones de equidad, etc., también es cierto que el precio que se acabe fijando puede generar ineficiencias considerables (ver Rothschild y White (1995)).

Aparentemente, cuanto mayor sea el coste de los estudios, mayor será la calidad esperada de la educación recibida (tanto en términos de formación como de relación de los estudios con la demanda que realiza el mercado ${ }^{8}$ ) y mayor la exigencia a los gestores. Los estudiantes podrían estar dispuestos a satisfacer unas mayores tasas y matrículas si se correspondiese con un mayor nivel de calidad en la educación y los servicios que reciben, siempre y cuando esto se refleje en unos mayores ingresos potenciales al acabar los estudios.

Supongamos, por ejemplo, que la función de ingresos netos de los que han estudiado dependa de un salario fijo $w$ (valor presente de los salarios futuros) que varía con el talento del estudiante-trabajador $\left(\varphi_{i}\right)$ y de la calidad de educación recibida en la universidad $k, H_{k}$ por la cual ha abonado unas tasas $T_{k}$,

$$
I N_{k}=w\left(1+\varphi_{i} H_{k}^{\varepsilon}\right)-T_{k}
$$

En esta situación, si el estudiante no estuviese sometido a restricciones presupuestarias o restricciones al crédito, escogería la universidad que ofreciese la mejor calidad a un menor precio (tasa), es decir, escogería aquella universidad que le permitiese maximizar su función de ingresos netos. Como es lógico, cada agente solo estudiaría si $\varphi_{i} H_{k}{ }^{k}-T_{k}>0, \forall k$ )

8 En el estudio de la FBBVA (2006) se indica que los estudiantes de las universidades privadas valoran mucho más que los de las universidades públicas la relación de la formación que reciben con la demanda que se realiza desde el mercado. Aspecto muy poco valorado por los estudiantes de las universidades públicas. 
Gráficamente, vemos cómo el mapa de curvas de indiferencia sería:

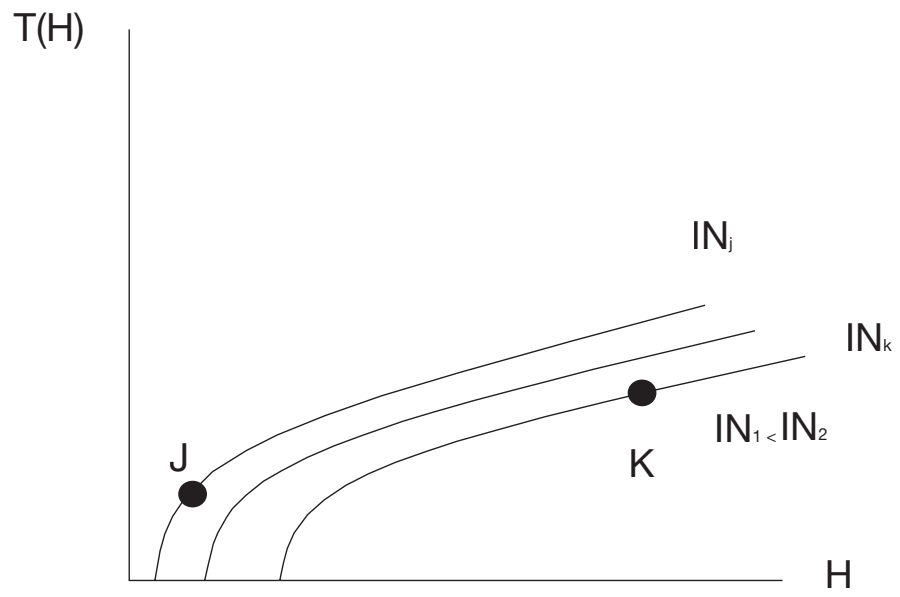

Como puede verse, comparando los puntos $K$ y $J$, un estudiante $i$ preferiría la universidad $k$ a la $j$, a pesar de que la primera fijase una tasa superior porque ésta ofrecería una mejor calidad, lo que le daría acceso a mayores salarios en el futuro. Además, cuanto más talento tuviese el estudiante, más estaría dispuesto a pagar por recibir una enseñanza de mayor calidad, puesto que su salario sería superior al del resto de estudiantes con menor talento.

Sin embargo, éste razonamiento no parece que se corresponda con la postura de los estudiantes, quienes en su mayoría no están dispuestos a asumir incrementos en el coste de las matrículas a cambio de mayor calidad ${ }^{9}$ (ver FBBVA 2006). Sería interesante analizar hasta qué punto la decisión de los estudiantes variaría si estas tasas se financiasen con instrumentos que permitiesen diferir el pago al futuro.

En el caso de que hubiese restricciones presupuestarias o de crédito, la decisión del estudiante no dependería de los recursos futuros (actualizados a traves de la deuda) sino de la disponibilidad inmediata. De ahí que cualquier incremento en las tasas sea muy importante en la decisión de los estudiantes. A iguales tasas escogería la universidad con mayor calidad, pero sólo podrá escoger la que le permita su renta inicial. Dado un nivel de renta inicial $Y_{0}$, a pesar de que $\mathrm{w} \varphi_{i} H_{k}{ }^{\varepsilon}-T_{k}>0, \forall k$ ) (el alumno podría cubrir el coste de la matrícula endeudándose), solo podría escoger aquella universidad con una tasa $T_{k}<Y_{0}$. De ahí la necesidad de que cualquier reforma en el modelo de tasas deba recoger un sistema de ayudas que permita no estar sometido a la restricción inicial.

Un argumento en contra de incrementar las tasas es que unas bajas tasas y matrículas académicas reducen considerablemente las restricciones en la demanda y esto

$9 \quad$ El $70 \%$ de los estudiantes encuestados se manifestaban en contra de permitir incrementos del $10 \%$ en las matrículas acompañados de incrementos en la calidad. 
debería permitir a las universidades seleccionar entre un mayor número de estudiantes y escoger a los mejores. Pero no parece que ésta sea la situación en la mayoría de estudios de la universidad española, en la cual predominan los estudios caracterizados por unas notas de corte muy bajas (extrañamente por encima del 5,5 y muchos de ellos sin nota de corte, especialmente en los estudios ofrecidos en los centros privados adscritos a las universidades públicas) y con una relación de demanda sobre plazas ofrecidas también muy bajas (difícilmente supera la relación de un solicitud en primera preferencia por plaza ofrecida, ver CRUE 2002 y MEC 2005).

Finalmente, no podemos olvidar que unas matrículas bajas no incentivan al estudiante a realizar un mayor esfuerzo porque el «fracaso» tiene un coste relativamente bajo. En este sentido, una mayor matrícula incrementa el coste de oportunidad de no esforzarse. De ahí quizá las penalizaciones que acostumbran a fijar las universidades para las segundas y siguientes matrículas.

\subsection{Tasas y calidad en la oferta de educación superior}

El análisis de la calidad de la oferta de educación superior y el sistema de financiación de las universidades ha recibido atención solamente desde hace unos pocos años. La mayoría de artículos en la literatura analizan en qué medida el modelo de financiación afecta a las decisiones de la universidad en cuanto a la gestión de la actividad docente y la actividad investigadora, aspectos que directa o indirectamente influyen sobre la calidad de la oferta educativa (ver Beath et al. (2005), Beath et al (2003), Brickley y Zimmerman (2001), Del Rey (2001), Del Rey y Romero (2004), Eple y Romano (1998), Fethke (2005), Kemnitz (2004), Vanhaecht y Pauwels (2005) y Rossello (2006 $)$, entre otros). En esta sección presentamos un modelo sobre estratificación de universidades y segregación de estudiantes por talento, desarrollado con más detalle en Rosselló (2006b).

Una política de tasas y matrículas bajas no tendría por qué afectar el volumen de recursos de que disponen las universidades, y con ello al nivel de calidad de la enseñanza, siempre que vayan acompañadas del nivel suficiente de transferencias por parte de la Administración.

Un primer problema con el modelo actual de transferencias basado en una contribución por estudiante (y sólo complementariamente en una aportación por los resultados en su actividad investigadora), sin relacionarla con la calidad de los estudios, con las tasas de éxito-fracaso de los estudiantes, los indicadores de demanda de los estudios, etc. provoca que los esfuerzos de la universidad se ciñan a encontrar el número suficiente de estudiantes ${ }^{10}$ para poder cubrir la oferta de plazas de que dis-

10 Esto quizá explica en parte la constante mejora en las facilidades que ofrecen las universidades para permitir prolongar la duración de los estudios. Nos referimos a la ampliación del número de convocatorias, a la reducción de los créditos mínimos a aprobar para continuar con los estudios, al coste de las segundas y terceras matrículas, etc. 
ponen, que en atraer solamente a los mejores, mejorar el modelo de gestión administrativa, diseñar un modelo adecuado de asignación de recursos entre Departamentos (hoy en día regido básicamente también por el número de estudiantes), contratar a los mejores profesores e investigadores, etc. En definitiva, el modelo actual no incentiva que las universidades compitan entre ellas, no solamente en oferta de plazas, sino en términos de calidad y de precios.

Un segundo problema reside en las restricciones presupuestarias a las que están sujetas las propias administraciones. Si la calidad de la educación que puede ofrecer una universidad depende de su nivel de ingresos y el modelo de financiación se basa en las transferencias de las administraciones, la calidad de la educación ofrecida en las universidades acaba dependiendo del volumen de las mismas.

Cabe pensar que es posible que un modelo en el cual las administraciones deciden las tasas que pagan los estudiantes y éstas son uniformes en todas las universidades de su jurisdicción, independientemente del talento y del nivel de renta de los estudiantes, y de los costes de las universidades, evite la estratificación de las universidades y la segregación de los estudiantes (problemas que son empíricamente demostrados en Hoxby (1997) y Epple y Romano (2003) en modelos competitivos con precios libres). Sin embargo, esta uniformidad en la calidad podría conseguirse con el coste de fijar límites al nivel de calidad que pueden ofrecer las mejores universidades y supondría, de hecho, una subvención a las universidades menos eficientes.

Esta situación queda ilustrada en un modelo de competencia entre universidades públicas en el cual suponemos que la calidad de la enseñanza que ofrecen las universidades, $H$, depende de la calidad investigadora del conjunto de profesores $(\mu)$ y del talento medio de los alumnos $(\varphi)$ siguiendo

$$
H_{k}=A_{k} \bar{\mu}_{k}^{\alpha} \bar{\varphi}_{k}^{l-\alpha}
$$

$A_{k}$ representa un conjunto de características de la universidad (modelo de gestión, infraestructuras, acceso a la tecnología, etc.) que afectan a la calidad de la enseñanza que ofrece.

El objetivo de la universidad es maximizar el nivel de calidad que ofrece, y para ello debe escoger el talento medio de profesores y estudiantes — los inputs- sujeto a una restricción presupuestaria (sin permitir el recurso al endeudamiento) que sigue:

$$
T(H) M_{k}=N_{k} W\left[1+w\left(\mu_{k}^{-},\right)\right]+\mathrm{c}\left(\varphi_{k}\right)
$$

donde $w\left(\bar{\mu}_{k}\right)$ representa los complementos de investigación que reciben los profesores (que suponemos que no se calculan individualmente), que se agregan a un salario $\mathrm{W}$ igual para todos los profesores de cualquier universidad; por otro lado, $w\left(\bar{\varphi}_{k}\right)$ representa en qué medida la calidad de los alumnos repercute en los costes de la provisión del servicio. Estos costes podrían justificarse como todo aquello que la universidad debería ofrecer, como servicios para atraer a los mejores estudiantes, que también intentan ser atraidos por otras universidades (becas, servicios de orientación, instalaciones deportivas y de ocio, etc.). 
Para determinadas formas funcionales $\left[w\left(\bar{u}_{k}\right)=\eta_{k} \bar{\mu}_{k}\right.$ y $\left.c\left({ }^{\alpha} \bar{\varphi}_{k k}\right)=\rho_{k}{ }^{\alpha} \bar{\varphi}_{k k}\right]$ y después de los cálculos pertinentes ${ }^{11}$, los autores encuentran una oferta de calidad $(H)$ de la educación para cada universidad $k$ que, en equilibrio, depende de los costes salariales del profesorado, de los costes que tiene la universidad para atraer a los mejores estudiantes, del número de estudiantes $(M)$ y profesores $(N)$ que se consideran fijos, pero también de los ingresos por estudiante que recibe la universidad. Éstos provienen a su vez de las transferencias de la administración ( $t s$ ) y de los ingresos por matrículas ( $t x$, fijadas también desde la Administración). Finalmente, la oferta de calidad también depende de un conjunto de variables, recogidas en el término A, que representa las dotaciones en infraestructuras, el modelo de gestión de cada universidad, etc.

Así pues, la función de oferta de calidad sería,

$$
T(H)^{s}=W \frac{N_{k}}{M_{k}}\left[H_{k} \frac{1}{A_{k}}\left(\frac{\rho_{k}}{\alpha}\right)^{\alpha}\left(\frac{\eta_{k}}{1-\alpha}\right)^{1-\alpha}\left(\frac{1}{N_{k} W}\right)^{\alpha}+1\right]
$$

con los ingresos de la universidad que siguen $T(H)=t x+t s$. El parámetro $\eta$ se determinaría en el mercado de profesores, que no se analiza aquí, y $\rho$ podría considerarse como exógeno.

Gráficamente, podemos representar la oferta de calidad en equilibrio:

Este modelo, una vez introducimos el comportamiento de los propios estudiantes ${ }^{12}$, como se ha definido en la sección anterior y bajo algunas restricciones, permite derivar algunos resultados interesantes. En primer lugar, si la administración decisiese no subvencionar a ninguna universidad ( $\sin$ ayudas, $t s=0$ ) y fijase unas tasa homogénea para todos los estudiantes ( $t x)$, se daría el fenómeno de la estratificación de las universidades (si fuesen distintas, cada una ofrecería un nivel distinto de calidad con los mismos ingresos) y los estudiantes se concentrarían en una u otra universidad dependiendo de su talento (bajo el supuesto de que éstos difieren en talento y no están sujetos a restricciones presupuestarias). El nivel de calidad de la oferta educativa de cada universidad dependería del nivel de la tasa que fijase la administración $(H(t x))$ y de su estructura de costes.

En segundo lugar, si la administración pretendiese que las universidades ofreciesen la misma calidad, ésta podría decidir transferir una cantidad por estudiante $(t s)$ dirigida a las universidades que para ofrecer el mismo nivel de calidad que el resto deberían afrontar mayores costes. Un primer problema de esta política es que de hecho se estaría subvencionando a las universidades menos eficientes. Un segundo problema es que la administración tiene recursos limitados $(G)$, esto hace que finalmente que la calidad que puede ofrecer la mejor de las universidades dependa de la cantidad de

11 Ver Rosselló 2006b para una descripción más detallada del modelo y de los resultados.

12 También deberíamo definir el proceso de selección de las universidades. En el caso español, recordemos que éstas no seleccionan a sus estudiantes puesto que se sigue un procedimiento automático que asigna a los estudiantes entre las universidades teniendo el cuenta el orden de preferencia, el talento del estudiante, el número de plazas, etc. 


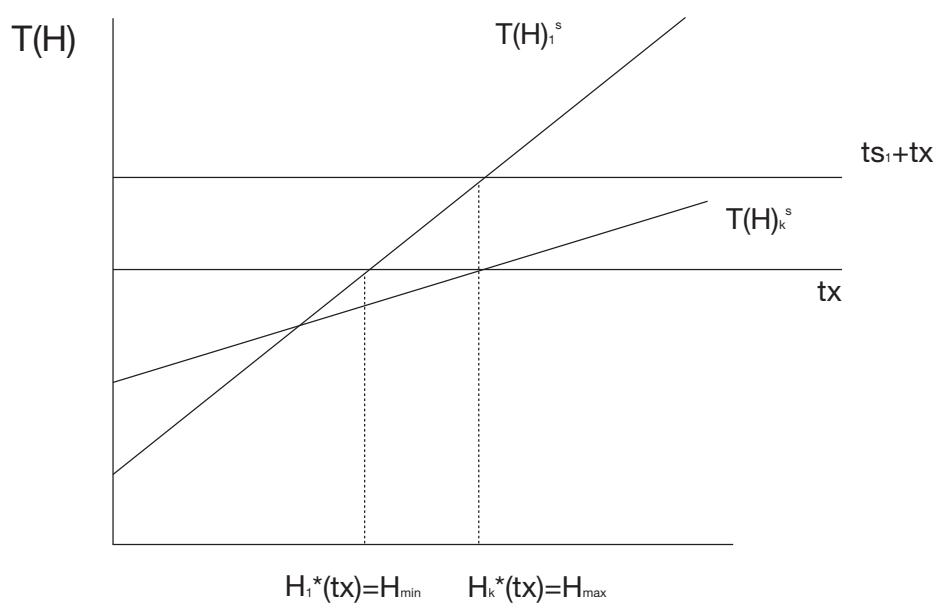

recursos que está dispuesta a ofrecer la administración y de las diferencias que existen entre universidades. Cuanto mayores son las tasas, mayor sería la diferencia en la calidad de la educación ofrecida en cada universidad. Corregir estas diferencias requeriría de mayores recursos por parte de la administración. En cambio, manteniendo bajas las tasas es posible mantener diferencias muy reducidas en los niveles de calidad, y esto requiere de bajos volúmenes de recursos para compensar una u otra universidad, a costa de reducir el nivel de calidad del conjunto de universidades.

Así pues, en equilibrio, las tasas que podrían cobrarse a todos los estudiantes por igual, y con ello el nivel de calidad de la enseñanza, dependen del nivel de recursos disponibles (G). En particular, las tasas que podrían fijarse en el sistema universitario para que garantizasen una homogeneidad en la calidad de la universidad, dados unos recursos presupuestarios seguiría

$$
t x=f\left(\stackrel{+}{G}, \stackrel{+}{M}_{l}, \bar{M}_{k}, \frac{\bar{\rho}_{l}}{\rho k}, \frac{\bar{\eta}_{l}}{\eta k}, \frac{\bar{A}_{k}}{A_{l}}\right)
$$

Cabe señalar, sin embargo, que la política de tasas subvencionadas en un modelo competitivo corregiría la estratificación de las universidades, pero no nos dice nada sobre la segregación de los estudiantes, puesto que éstos estarían indiferentes entre acudir a una u otra universidad, porque ambas ofrecen la misma calidad al mismo coste.

Así pues, el modelo actual de tasas uniformes y subvencionadas por la administración da como resultado una oferta uniforme en cuanto al nivel de calidad de la oferta educativa, resultado opuesto al modelo de diversificación en la oferta que funciona en los Estados Unidos, incluso entre las mismas universidades públicas.

Finalmente, un problema adicional de este modelo basado en las transferencias desde la Administración es que acentúa la dependencia de las universidades respecto de las Administraciones. Si bien en una estructura descentralizada, como sucede con las 
universidades como entes autónomos, lo racional sería transferir los recursos a las universidades — condicionados quizá a la consecución de objetivos de calidad- y que éstas decidiesen su política de incentivos, la contratación del profesorado, la selección de estudiantes, etc, en España la Administración opta por una normativa de tipo preventivo que restringe la autonomía de las universidades a la hora de seleccionar alumnos, de seleccionar y escoger al profesorado, etc. (ver el último informe de la FCyD 2005 al respecto) que no se acompaña de evaluaciones ex-post de calidad en la enseñanza, o de análisis de eficacia-eficiencia en la gestión de las universidades. Así, un mayor grado de autonomía tampoco es garantía de una gestión de mayor calidad, si no se definen correctamente los incentivos o penalizaciones, eminentemente en términos de financiación, por esa gestión.

\section{Alternativas al modelo de transferencias públicas.}

La observación de las tendencias que siguen otros países de la OCDE en cuanto al modelo de provisión de la enseñanza superior muestra cómo la alternativa al modelo actual no pasa por eliminar las transferencias desde la administración, sino por reducir su peso y aumentar el peso de los recursos que proceden del sector privado. Incluso en países como Estados Unidos, la mayoría de universidades públicas siguen siendo muy dependientes de las transferencias de la Administración.

En el ámbito europeo algunos países ya se han planteado modificar el modelo de financiación, aunque todavía, a excepción del Reino Unido, no lo han hecho, manteniendo un modelo de transferencias a las universidades que permite mantener el coste de los estudios muy por debajo del coste de provisión, sin permitir el aumento de las matrículas — en algunos casos, como en Alemania, la matrícula es totalmente gratuita-. En España, aunque las tasas y matrículas siguen manteniéndose bajas, y así será con los nuevos grados, se han introducido, aunque tímidamente, nuevos instrumentos de financiación como los créditos a los estudiantes.

Sin embargo, la creciente demanda de recursos por parte de la universidad, unida a las propias restricciones financieras de las administraciones, hacen pensar que a medio plazo deberá avanzarse en la reforma del sistema de financiación de las universidades. Es obvio que incrementar las tasas y las matrículas que se cobran a los estudiantes es una de las posibilidades que deberían barajarse. En este sentido, cabe tener en cuenta que la modificación en el sistema de financiación de las universidades debería ir estrechamente unida a una reforma en el sistema de becas y ayudas.

Aunque cualquier modificación en el sistema de universidades públicas se hará de acuerdo con las particularidades de cada país, un modelo de universidad pública que merece recibir atención es el de Estados Unidos, tanto por su proximidad a un modelo de gestión privada de universidad como a la experiencia en ese país en el diseño de instru- 
mentos financieros dirigidos a los estudiantes. En las páginas que siguen analizamos el modelo de financiación de las universidades públicas que se aplica en Estados Unidos, así como las distintas reformas que se han introducido en los modelos británico y australiano.

\subsection{Algunos aspectos del sistema público de ayudas a la educación superior en Estados Unidos ${ }^{13}$}

En Estados Unidos el debate sobre la deseabilidad de la provisión pública de la educación superior, así como el actual modelo que funciona, han venido marcados por el papel subsidiario que se atribuye a la administración pública en aquellos sectores en los que el sector privado puede desarrollarse. Es por ello que la provisión pública de la educación superior se diseña en base a tres premisas: i) garantizar el acceso al mayor número de personas para aprovechar las externalidades derivadas de la educación superior, ii) corregir el componente regresivo ligado a la provisión pública de la educación superior (bien sea por comparación con los que no realizan estudios de educación superior, bien por comparación con los estudiantes por sus niveles de renta), iii) corregir las ineficiencias propias de un sistema público de provisión (referido al modelo de gestión de las universidades, al rendimientos de los propios estudiantes, etc.)

De ahí que el modelo de financiación haya tendido a reducir la dependencia de las universidades públicas respecto de las transferencias públicas, atribuyendo más peso a las tasas y matrículas, descentralizando la decisión sobre éstas a las universidades y fomentando la competencia entre ellas. Así mismo, el modelo de ayudas y becas destinadas a los estudiantes ha ido modificándose con el objetivo de permitir el acceso a todos los estudiantes sin recursos (aunque sea a través de diferir el pago de los estudios al futuro) y a maximizar el rendimiento de los estudiantes. Es decir, es un modelo que se basa en permitir unas mayores tasas pero también asigna un mayor volumen de recursos a ayudas y becas (este hecho es especialmente notable en las universidades privadas, como puede observarse en la figura1).

El gasto público en educación se articula, como en otros países, en dos grandes bloques. Por un lado, el volumen de transferencias que reciben directamente las universidades, que permiten que éstas no trasladen el coste de la educación enteramente a los estudiantes. En segundo lugar, aquellos programas e iniciativas dirigidos a los estudiantes. Cabe señalar que, a pesar de que cada uno de los Estados tiene su propia política universitaria, la mayor parte de los recursos destinados a ayudas y becas proviene del gobierno federal (en una relación de 1 a 13 en 2004 según el College Board). Adicionalmente, también cabe tener en cuenta que las propias universidades, en particular los centros privados, disponen de recursos para destinar a becas y ayudas.

13 Ver Bowen et al, cap. 8, y College Board 2005a y Minnesota Office for Higher Education (http://www.mheso.state.mn.us) 


\section{FIGURA 1}

\section{COSTE DE LOS ESTUDIOS UNIVERSITARIOS ANTES Y DESPUÉS DE AYUDAS Y BECAS. EE.UU, 1995-2005}

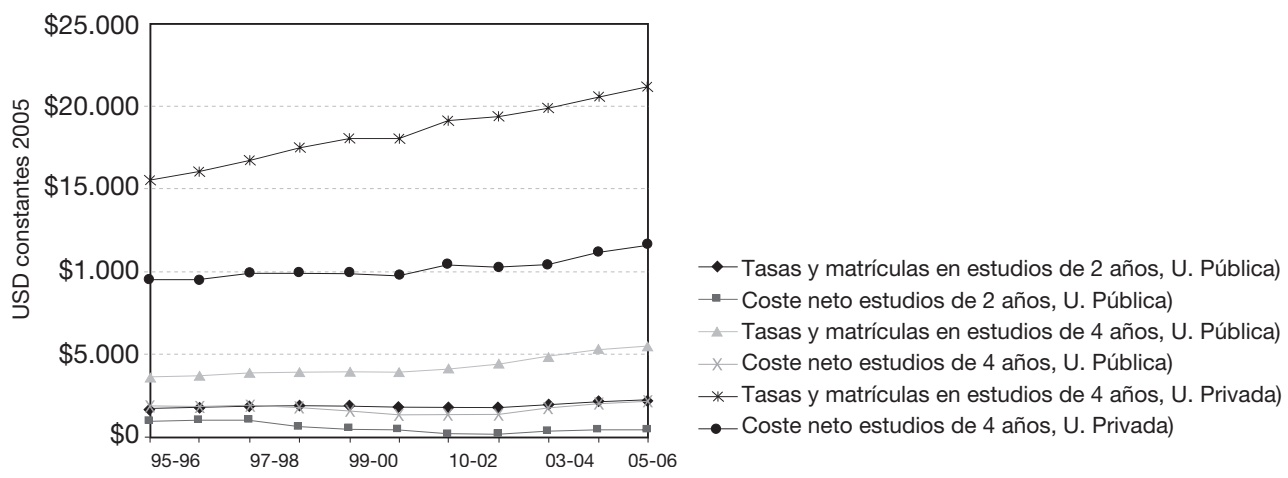

Fuente: College Board 2005

En lo que se refiere al sistema público de becas y ayudas, hay tres tipos de instrumentos: i) las becas a los estudiantes ${ }^{14}$, ii) los créditos ${ }^{15}$, iii) los créditos y las deducciones fiscales (incluyendo los descuentos por aportaciones a cuentas de ahorro cuyos recursos se dirijan a financiar los estudios).

En cuanto al sistema de becas, hay dos tipos de instrumentos ${ }^{16}$. En primer lugar, las ayudas basadas en el nivel de renta de los estudiantes (need-based aid), parecidas al modelo de becas que funciona en España. La diferencia con las becas en España es que este programa solamente cubre gastos de tasas y matrículas, no gastos de residencia y desplazamiento, y además la ayuda ${ }^{17}$ se decide a partir de una estimación de la disponibilidad financiera de los padres, considerándose no solamente el nivel de ingresos sino todos los activos, y del coste de los estudios (tasas y matrículas, alojamiento, libros, etc.). En segundo lugar, están las llamadas merit-aid grants, que puede recibir cualquier estudiante con talento, independientemente de su nivel de renta. Este tipo de ayuda acostumbran a ofrecerlas las propias universidades, y es utilizado para atraer a estudiantes de gran talento que generalmente son pretendidos por otras universidades.

En cuanto al sistema de ayudas, en los años 90, ante el incremento en los costes de la enseñanza superior, hubo una gran presión para aumentar el nivel de las ayudas del programa Pell Grants. Los detractores de esta medida argumentaban que esto sólo comportaría incrementos sucesivos en las tasas, de forma que los estudiantes con me-

14 El programa se denomina Pell Grants y se complementa con el Federal Supplemental Educational Opportunity Grant.

15 Federal Stafford Loans.

16 Hay otros programas como las becas para atletas o las becas de trabajo, que deciden cada una de las universidades.

17 Hay un máximo al volumen de la beca que puede recibirse, entre los 400 y los 4.050USD en 2004-2005) 
nos recursos no se beneficiarían del incremento en la dotaciones de recursos. Para evitar esa posibilidad se crearon dos instrumentos alternativos.

En primer lugar, siempre en un entorno con tasas liberalizadas, el Gobierno Federal fomentó el uso intensivo de los créditos a los estudiantes ${ }^{18}$, dándose — ver figura 2- un desvío significativo de recursos en términos reales del programa de becas hacia este nuevo instrumento.

FIGURA 2

\% DE AYUDAS FEDERALES DESTINADAS A BECAS, CRÉDITOS Y AYUDAS FISCALES. 1963-2005 (DÓLARES 2004)

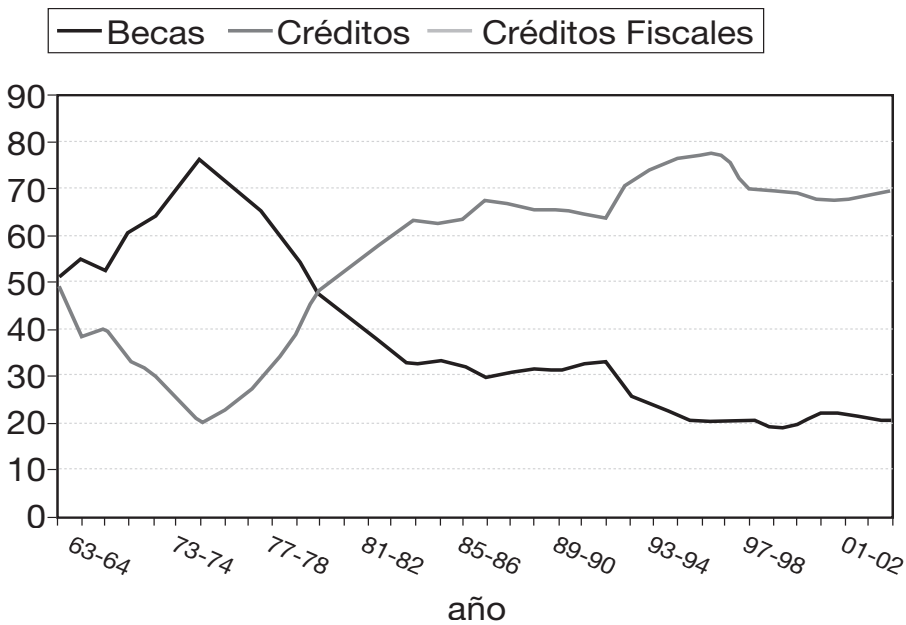

Fuente: College Board 2005

La crítica a este tipo de medida es que las familias con menores recursos ${ }^{19}$ se benefician escasamente de este recurso. En cambio, se beneficia a las familias con ingresos medios y altos, que aún sin estos recursos podrían permitirse mandar a sus hijos a la universidad.

En segundo lugar, a mediados de los 90 se introdujeron las deducciones y los créditos fiscales ${ }^{20}$, que solamente permiten cubrir gastos de tasas y matrículas. Hay dos modalidades a las que pueden acogerse los padres, o los propios estudiantes, generando cada una efectos redistributivos distintos en cada colectivo. Por un lado, como gasto deducible en la cuota del impuesto sobre la renta con un máximo ${ }^{21}$. Por otro lado,

18 Estos créditos se agrupan en distintos programas: Federal Perkins Loan, Federal Stafford Loans, Federal Direct Stafford Loans, Federal Parent Loans for Undergraduate Students (PLUS), Federal Health Professions Programs.

19 También hay límites máximos al volumen de crédito que pueden subscribir y que solamente en algunos casos permiten cubrir los costes de matrícula y tasas en una universidad de élite.

20 Otros programas son el Tuition and Fees Deduction, Employer Provided Educational Assistance.

21 Hope Tax Credit, con un máximo de 1.500 USD 
se ofrece la posibilidad de un descuento en la base ${ }^{22}$, con lo cual la deducción real dependerá del tipo marginal de los progenitores. En caso de que haya gastos de manutención y desplazamiento la carga financiera que recaería sobre los estudiantes sería muy grande. Finalmente, existe la posibilidad de descontarse fiscalmente los intereses de los créditos en los impuestos federales ${ }^{23}$.

Es decir, de forma parecida al modelo que funciona en la mayoría de países europeos, la universidad pública en EE.UU también se basa de forma importante en la transferencia de recursos publicos. Sin embargo, el peso de éstos disminuye constantemente, y las tasas y matrículas que pagan los estudiantes son muy superiores, en promedio, a las que fijan las universidades públicas europeas. Esto indica que las administraciones públicas en Estados Unidos actualmente se decantan más por aportar directamente recursos a los estudiantes, que después recuperan, en contraposición a las transferencias directas a las universidades para permitir mantener unas tasas y matrículas bajas.

De esta forma este modelo tiene al menos dos aspectos positivos desde el punto de vista de la eficiencia y la equidad. Por un lado, el hecho de que las universidades puedan fijar tasas más elevadas no limita tanto la capacidad de generación de recursos de éstas (aunque los ingresos por matrículas siguen siendo poco importantes en el conjunto de ingresos) y las incentiva a competir en «precios» para atraer a los mejores estudiantes (en comparación con el modelo europeo de tasas y matrículas uniformes). Por otro lado, permite el acceso a los estudiantes sin recursos sin reducir los incentivos de éstos a realizar un mayor esfuerzo, dado que el coste de los estudios recae siempre sobre el estudiante, aunque se financien con créditos, con lo cual también se corrige el sesgo regresivo derivado de la financiación de la educación superior.

\section{Calidad de la enseñanza en las universidades públicas en Estados Unidos:} la estratificación, ¿defecto o virtud?

La característica principal del modelo universitario en EE.UU es la diversificación en la oferta de centros universitarios, que permite a los estudiantes escoger los centros que mejor se ajusten a sus capacidades (de talento y renta) y preferencias. Sin embargo, la diversificación también va acompañada de una clara estratificación de las universidades por la calidad de su oferta y también de una segregación de los estudiantes en cuanto a su talento y nivel de renta.

La estratificación la encontramos incluso entre los centros públicos, y ésta viene determinada en parte por el volumen de recursos que los estados dedican a la política universitaria y la capacidad que éstas han mostrado de gestionar los recursos (que incluye la capacidad de atraer recursos del sector privado). Así pues nos encontramos con un modelo en el que son las propias administraciones las que incentivan la competencia entre universidades. 
Por un lado, hay un número importante de universidades públicas que operan exclusivamente en el ámbito regional. Éstas están muy orientadas hacia la formación y tienen un vínculo muy estrecho con el mercado de trabajo local-regional. Las matrículas en estas universidades acostumbran a estar muy por debajo de las universidades públicas de élite que están localizadas en el mismo Estado. De ahí que las universidades de ámbito regional concentren la mayoría de beneficiarios del programa Pell Grants $^{24}$. Al igual que sucede en algunas universidades públicas europeas, un número importante de estas universidades se caracterizan por sus altas tasas de fracaso y abandono, la baja calidad en la oferta educativa, elevasas tasas de paro de los estudiantes, derivados en parte de problemas de finaciación.

Por otro lado, están las universidades públicas de élite, cuya matrícula acostumbra a ser considerablemente más elevada y destacan, aunque sólamente algunas, por su competitividad y su calidad, tanto en el ámbito de la docencia como de la investigación.

Así pues, en cierta medida la estratificación en las universidades va acompañada de cierto nivel de segregación de los estudiantes por nivel de talento y renta (Hoxby (1997) y Epple y Romano (2003)), que no es corregido por el actual sistema de becas y ayudas. Hoxby (1997) muestra cómo el problema está en que los estudiantes con menos recursos, independientemente de su talento, solicitan en menor medida el acceso a universidades de élite, indicando que ex-ante se produce una autoselección de los estudiantes. De esta forma, el sistema de becas y ayudas no es suficiente para garantizar la igualdad de oportunidades en el acceso a la educación superior ${ }^{25}$.

\subsection{Algunos aspectos de la reforma del sistema público de ayudas a la educación superior en el Reino Unido ${ }^{26}$}

En la Unión Europea encontramos modelos muy distintos de universidad pública. Así, en Holanda, Italia ${ }^{27}$ y España la enseñanza superior actualmente ya no es gratuita, a pesar de que las matrículas son en general muy bajas y de momento no hay indicios de que esta situación vaya a cambiar. En cambio, en países como Francia o Alemania, la enseñanza es gratuita ${ }^{28}$, y a corto plazo no hay prevista ninguna modificación que permita cambiar esta situación en las universidades públicas.

Sin embargo, desde hace unos pocos años, en el Reino Unido comenzaron a introducirse una serie de reformas dirigidas a permitir que las universidades puedan aplicar incrementos substanciales en el coste de las matrículas de los estudiantes, has-

24 En comparación con las universidades pública o privadas de élite la Pell Grant no llegaría a cubrir el $20 \%$ del coste de la matrícula

25 Sin embargo, los estudiantes con mayor talento y menos renta también pueden optar a las becas (merit-based aid) de las mejores universidades públicas y de las universidades privadas (las cuales generalmente ofrecen becas y ayudas de mayor cuantía).

26 Ver Department of Skills and Education 2003a , 2003b, 2003c.

27 En Italia la matrícula depende de las circunstancias familiares de los estudiantes

28 En Alemania sigue vigente la prohibición de cobrar matrículas en la enseñanza superior. 
ta un máximo de 3.000 libras anuales. Este cambio tan significativo tiene algunas particularidades, dirigidas a corregir los efectos que podría generar un incremento de las tasas sobre el número de matriculados.

En primer lugar, las universidades británicas que se acojan a la reforma podrán fijar matrículas distintas según el tipo de estudios o el tipo cursos que quieran realizarse en esa universidad. De esta forma, las universidades podrán fijar las matrículas acordes con el coste de los estudios, y en algunos casos podrán financiar las titulacones que consideren importantes pero cuyo interés o demanda exigen matrículas inferiores al coste de provisión. Esta opción se impuso frente a la de incrementar las tasas aunque sin permitir la heterogeneidad de las mismas.

En segundo lugar, aquellas universidades que fijen una matrícula superior al coste actual de las matrículas (1.125 libras esterlinas) deberán presentar una propuesta de financiación de los estudios para garantizar el acceso a aquellos estudiantes con menores recursos ${ }^{29}$. La administración transferirá el importe de las matrículas a las universidades y los estudiantes lo financiarán con créditos sin interés de la administración (descartándose la opción de financiarlo a través de un impuesto específico), que deberán pagar una vez hayan finalizado los estudios. En cualquier caso, los estudiantes no deberán abonar las matrículas al ingreso, sino al final de los estudios, siempre y cuando tengan ingresos superiores a las 15.000 libras esterlinas.

En tercer lugar, los ingresos que obtengan por esos incrementos deberán ser adicionales para las universidades. La administración no reducirá las aportaciones-transferencias que realizaba a esa universidad.

Finalmente, el nuevo modelo insistirá en la mejora de la información accesible a los estudiantes, en particular la información comparada entre distintas universidades, precios y calidad de los cursos, salidas profesionales, etc. Todo ello con el objetivo de mejorar capacidad de selección de los estudiantes.

\subsection{Algunos aspectos de la reforma del sistema público de ayudas a la educación superior en Australia.}

En Australia el modelo de financiación universitaria comenzó con el establecimiento de la Higher Education Contribution Scheme en el marco de la Higher Education Funding Act en 1988. En 1997 se introdujo la posibilidad de diferenciar tasas por estudios y actualmente se permite que las universidades incrementen las tasas de forma mucho más flexible (puede haber ciertas diferencias entre instituciones).

Los estudiantes no deben hacer frente a las matrículas al iniciar los estudios sino que deben hacer frente a dicho coste al graduarse, a través del sistema impositivo. En este sentido, los estudiantes graduados comienzan a hacer frente al pago de los costes de su educación siempre que sus ingresos superen los 25.000AusD. Además, la can-

29 Los estudiantes con menores recursos seguirían teniendo acceso a una beca de 1.000 libras (Higher Education Grant) y a la gratuidad de la matrícula hasta las 1.125 libras esterlinas. 
tidad que se paga depende del volumen de ingresos. Así, para ingresos entre 25.000 y 46.000AusD la contribución representará el 3\% de todos los ingresos, mientras que para ingresos superiores la contribución será del 6\% (la contribución se realiza hasta cubrir el coste de los estudios).

Las tasas repercutidas sobre los estudiantes no pueden superar el equivalente a un $25 \%$ del coste, y deben reflejar las diferencias entre unos y otros así como el potencial diferencial de ingresos. Se distinguen tres tipos de estudios : i) Humanidades y Ciencias Sociales (tasas bajas, entre \$0-5.010\$), ii) Matemáticas e Ingenierias (nivel medio, 0\$-7.137\$), iii) Ciencias Jurídicas, Odontología, Medicina, Veterinaria, etc. (tasas altas, $0 \$$ y 8.355).

En 2005 se permitió a las universidades fijar tasas diferenciadas según el tipo de estudios, con un máximo a nivel nacional (no hay una libertad total a la hora de decidir las tasas ni entre universidades ni entre estudios).

Así pues, las diferencias del modelo australiano respecto al británico residen en el sistema de financiación a los estudiantes. Si bien las tasas pueden llegar a cubrir el $25 \%$ del coste estimado de los estudios, los estudiantes con recursos pueden pagar ese coste, con descuentos, en el momento de la matrícula. En caso de no disponer de recursos suficientes, pueden acogerse al Student Financial Supplement Écheme, que funciona como un crédito que los estudiantes deberán amortizar desde el momento de finalizar los estudios (siempre y cuando tengan unos ingresos superiores a los 30.000 AusD en 2005). De momento no hay programas de becas para estudiantes, todas las ayudas públicas se dan en la forma de créditos (que también recogen una cantidad para cubrir los gastos por desplazamiento, alojamiento y manutención).

Otro de los problemas que se plantean se refiere al impacto del incremento de las matrículas sobre el número de matriculados en educación superior. De momento, los datos parecen indicar que desde que se estableció el sistema diferenciado de tasas y desde que las universidades tienen mayor flexilibilidad a la hora de decidir las mismas, el volumen de matriculados en educación superior ha aumentado. Además, este resultado se ha observado en todos los grupos sociales.

\section{Conclusiones}

En este artículo hemos analizado los efectos que el actual sistema de financiación de las universidades tiene sobre la calidad de la oferta educativa. En particular, hemos mostrado que incluso en un modelo de universidades públicas podría darse el fenómeno de la estratificación de las universidades (aparición de universidades de élite) y la segregación de los estudiantes por su talento.

En segundo lugar, hemos mostrado cómo una política orientada a evitar la estratificación podría consistir en fijar tasas uniformes y bajas, garantizando que todos los estudiantes tienen la misma oportunidad de acceso, y subvencionar a las universidades con mayores costes. Esta política es de hecho la que observamos en España, donde universidades de la misma región establecen el mismo nivel de tasas para el mismo tipo de estudios, independientemente de la estructura de costes de cada universidad. 
Los efectos de este tipo de iniciativas son importantes desde el punto de vista de la calidad de la oferta. Por un lado, se estaría subvencionando a las universidades más ineficientes. Por otro lado, la calidad de la oferta de todo el sistema (todas ofrecerían la misma calidad) dependería de la cantidad de recursos que la administración estuviese dispuesta a dedicar al sector. De esta forma, esta política, en caso de restricciones presupuestarias por parte de las administraciones, limitaría incluso el nivel máximo de calidad que podrían ofrecer las universidades más eficientes. En este sentido, cabe tener en cuenta que los estudiantes que estuviesen dispuestos a pagar tasas más elevadas para adquirir una formación de mayor calidad no encontrarían esa oferta.

Un segundo aspecto que cabe señalar es que es necesario diferenciar entre el problema de la financiación de las universidades y el problema de los estudiantes. En este sentido, una reforma que permita incrementos en las tasas, esto es, que se traslade una mayor parte del coste de los estudios de las administraciones a las familias, no tiene por qué representar, tal y como se ha visto en otros países como Canadá, Estados Unidos, Australia, etc, una disminución en el número de estudiantes, ni siquiera para los estratos más bajos de renta. Esto dependerá en parte de los instrumentos - ayudas y becas- que se establezcan para permitir el acceso a los estudiantes con menos recursos, sin que ello quiera decir que la enseñanza les resultará gratuita. De esta forma se consigue corregir los problemas de inequidad del actual modelo de tasas uniformes y subvencionadas y las ineficientes derivadas del mismo modelo.

\section{Referencias bibliográficas}

[1] AVERY, C., y C. HOXBY (2004): «Do and should Financial Aid Packages affect Student's Choices?», en College Choices: The economics of where to go, when to go and how to pay for it, The university of Chicago Press, 239-302.

[2] BEATH, J., J. POYATO-THEOTOKY y D. ULPH, (2005): «University Funding Systems and their impact on Research and Teachng: A general Frameworks». Artículo presentado en la Conference on Higher Education, Multijurisdictionality and Globalisation, Mons (Bélgica)

[3] BEATH, J., R.F. OWEN, J. POYAGO-THEOTOKY y D. ULPH (2003): «Optimal incentives for income-generation in universities: the rule of thumb for the Compton tax», International Journal of Industrial Organization, 21, 1301-1322.

[4] BRICKLEY, J.A y J.L. ZIMMERMAN (2001): «Changing incentives in a multitask environment: evidence from a top-tier business school». Journal of Corporate Finance 7, 367-396.

[5] BOK, D (2003): Universities in the Marketplace: the commercialization of higher education. Princeton University Press.

[6] BOWEN, W.G. , M.A. KURZWEIL, y E. M. TOBIN (2005): Equity and excellence in american higher education. University of Virginia Press

[7] CHATTERTON, P. y J. GODDARD (2003): «The Response of Higher Education Institutions to regional needs.» En Economic Geography of Higher Education: Kno- 
wledge infrastructure and learning regions. R. Rutten, F. Boekema y Elsa Kuijpers (Eds.), Routledge.

[8] CIGNO, A, y LUPORINI, A. (2003). «Scholarships or student loans? Subsidizing higher education in the presence of moral hazard». CESifo WP No. 973.

[9] COLLEGE BOARD: (2005a). Trends in Higher Education Series, The Washington Office of the College Board.

[10] COLLEGE BOARD (2005b): Trends in Student Aid, The Washington Office of the College Board

[11] COOK, P.J. y R.H. FRANK (2003): «The Growing Concentration of Top Students at Elite Schools,» en C.T. Clotfelter and M. Rothschild, eds. Studies of Supply and Demand in Higher Education, Chicago: University of Chicago Press for the NBER.

[12] CRUE (2002), Información Académica, Productiva y Financiera de las Universidades Públicas Españolas (2000-2001).

[13] DE FRAJA, G. y E. IOSSA (2002), «Competition among Universities and the emergence of the elite institutions». Bulletin of Economic Research, 54:3,

[14] DEL REY, E y M.M. RACIONERO (2006), «Financing Schemes for Higher Education». WP No. 460. The Australian National University.

[15] DEL REY, E. y L. ROMERO (2004), «Competition between public and private universities: quality, prices and exams». Working Paper, Universidad Carlos III.

[16] DEL REY, E. (2001), «Teaching versus Research: A Model of State University Competition». Journal of Urban Economics 49, 356-373.

[17] DEPARTMENT FOR EDUCATION AND SKILLS (2003a), «Higher Education Funding: international comparisons». Gobierno del Reino Unido.

[18] DEPARTMENT FOR EDUCATION AND SKILLS (2003b),»Why not a fixed fee». Gobierno del Reino Unido.

[19] DEPARTMENT FOR EDUCATION AND SKILLS (2003c),»Why not a pure graduate tax?». Gobierno del Reino Unido.

[20] EPPLE, D., R. ROMANO y H. SIEG (2003), «Peer Effects, Financial Aid, and Selection of Students into Colleges and Universities: An Empirical Analysis.» Journal of Applied Econometrics, 18, 501-525.

[21] EPPLE, D., y R. ROMANO (1998): «Competition between Private and Public Schools, Vouchers, and Peer-Group Effects,» American Economic Review, 88(1), 33-62.

[22] EHREMBERG, R.G. y D. R. SHERMAN (1984): «Optimal Aid Policies for a selective university». Journal of Human Resources, 19 , 202-230

[23] EHRENBERG R.G. y M.J. RIZZO (2004). «Resident and non-resident tuition and enrollment at flagship state universities». In College Choices: The economics of where to go, when to go and how to pay for it. Caroline Hoxby (Ed), The university of Chicago Press, 303-354.

[24] FCyD (2005), Informe CYD 2005 sobre la contribución de las universidades españolas al desarrollo, Fundación Conocimiento y Desarrollo.

[25] FETHKE, G. (2005), «Strategic determination of higher education subsidies and tuitions». Economics of Education Review, 24, 601-609.

[26] FBBVA (2006): Estudio sobre la juventud universitaria española

[27] GARCÍA-PEÑALOSA, C. y K. WALDE, (2000), «Efficiency and equity effects of subsidies to higher education». Oxford Economic Papers 52. 
[28] GOLDIN, C., y L. KATZ (1998), «The Origins of State-Level Differences in the Public Provision of Higher Education: 1890-1940,» American Economic Review, 88, 303-308.

[29] GREENE, KENNETH V. (1994): «The public Choice of non-resident college tuition levels». Public Choice 78, 231-240

[30] HOXBY, C.M. (1997), «How the Changing Market Structure of U.S. Higher Education Explains College Tuition,» NBER working paper no. 6323.

[31] KEMNITZ, A. (2004), «Funding, Competition and Quality in Higher Education». Mimeo, University of Mannheim.

[32] KOSHAL, RAJINDAR K. y MANJULIKA KOSHAL (2000): « State Appropriation and Higher Education Tuition: What is the relationship?», Education Economics, 8(1), 81-89.

[33] MAS-COLELL, A. (2003), «The European Space of Higher Education: Incentive and Governance Issues». Revista di Politica Economica, 9-27.

[34] MCPHERSON, MICHAEL S. y M.O. SAPHIRO (1998): The Student Aid Game: meeting need and rewarding talent in american higher education. Princeton University Press.

[35] MEC Datos y Cifras del Sistema Universitario Español, varios años.

[36] ROSE, DAVID C. y ROBERT L. SORENSEN (1992): «High Tuition, financial aid, and cross-subsidization: do needy students really benefit?». Southern Economic Journal , 59(1), 66-76

[37] ROSSELLÓ, J. (2006a), »Incentives to Research Activities in European Public Universities», mimeo.

[38] ROSSELLÓ, J. (2006b), «Does a public university system prevent the stratification of public universities and the segregation of students?», mimeo.

[39] ROTHSCHILD, M. y L.J. WHITE (1995): «The Analytics of the Pricing of Higher Education and Other Services in Which the Customers Are Inputs.» Journal of Political Economy, 103, 573-586.

[40] VANHAECHT, E. y W. PAUWELS (2005), «University competition: symmetric or assymetric quality choices», mimeo, University of Antwerp.

[41] WINSTON, G.C. y D.J. ZIMMERMAN (2000), «Where is aggressive price competition taking higher education?», Change, 4, 10-18.

[42] WINSTON, G.C. (1999), «Subsidies, hierarchy and peers: the akward economics of higher education». Journal of Economic Perspectives, 13(1), 13-36. 
